\title{
DIODE LASER IRRADIATION COMBINED WITH PROPOLIS APPLICATION AS A TREATMENT FOR DENTINE HYPERSENSITIVITY
}

\author{
Polina I. Demydova \\ Department of Therapeutic Dentistry, Kharkiv National Medical University, Kharkiv, Ukraine
}

\begin{abstract}
InTroduction: Dentine hypersensitivity $(\mathrm{DH})$ is an extensive clinical condition, which is still of need to find a long-term treatment resulting in pain reduction.

ОвJеCTIVEs: The aim of this study was to evaluate and compare efficacy of sodium fluoride varnish application, diode laser $(810 \mathrm{~nm})$ irradiation, ethanolic extract of propolis application, and combined diode laser $(810 \mathrm{~nm})$ irradiation with ethanolic extract of propolis application for DH reduction in periodontal maintenance patients. MATERIAL AND METHODs: In total, 104 periodontal maintenance patients (416 teeth) completed this study. Dentine hypersensitivity was assessed using visual analogue scale (VAS). The patients were randomly divided into four groups according to the treatment: 1 group - diode laser $(810 \mathrm{~nm})$ irradiation, 2 - application of ethanolic extract of propolis, 3 - application of ethanolic extract of propolis, followed by diode laser $(810 \mathrm{~nm})$ irradiation, and $4^{\text {th }}$ group - sodium fluoride varnish application. The treated teeth were evaluated after treatment session and at one week, one month, three months, and six months post-operatively. The evaluations were analyzed using IBM SPSS Statistics, version 25.0 (IBM Corp., Armonk, NY, USA). Mean and standard deviation were calculated for each group. Wilcoxon's rank sum test and Friedman's test were used, and values of $p<0.05$ were considered as statistically significant.

RESULTS: All four groups showed a significant reduction in the VAS scores immediately after treatment. The most long-term results were obtained in group with combined treatment.

ConcLusions: The present study demonstrates that better result has been noted in group with combined application of ethanolic extract of propolis with diode laser $(810 \mathrm{~nm})$ irradiation.
\end{abstract}

KEY WORDS: propolis, LLLT, lasers, dentine sensitivity.

J Stoma 2020; 73, 4: 170-175

DOI: https://doi.org/10.5114/jos.2020.98312

\section{INTRODUCTION}

Dentine hypersensitivity (DH) is a common clinical finding, which has been defined as "a short, sharp pain arising from exposed dentine in response to stimuli typically thermal, evaporative, tactile, osmotic, or chemical, and which cannot be ascribed to any other form of dental pathology" $[1,2]$. Depending on the study, $\mathrm{DH}$ is a pervasive condition with a prevalence ranging from $3 \%$ to $57 \%$ of the general population. It is estimated to affect $15 \%$ of adult population, mostly within 30 and 40 years of age. It is known that women are affected more than men $[3,4]$.

\section{JOURNAL OF} STOMATOLOGY CZASOPISMO STOMATOLOGICZNE

AdDress For CORRespondence: Polina Demydova, Department of Therapeutic Dentistry, Kharkiv National Medical University, Nauky Ave, 4, Kharkiv, Ukraine, 61022, e-mail: polinademidovva@gmail.com 
Etiology of DH includes multiple factors. Various aspects, such as recession of gingiva, enamel loss, and tooth wear as well as patients' habits could be associated with DH. Moreover, frequent etiological factors are related to periodontal disease and consequences of its treatment. Chronic periodontitis is characterized by formation of periodontal pockets and alveolar bone resorption. As known in physiological conditions, enamel normally covers the dentine in the crown and cementum covers it in the root, which makes the tooth insensitive to direct stimulation. Due to chronic periodontitis and its treatment, such as scaling and root planning, the dentine might be uncovered. Consequently, DH occurs with an uncovered exposure of diameters of dentinal tubules $[5,6]$.

The mechanism of DH-related pain is described by several theories. Most widely acknowledged hypothesis is the so-called 'hydrodynamic theory'. In the beginning of the twentieth century, Alfred Gysi, without any scientific evidence, determined that there is an outward fluid flow inside dentinal tubules. Gysi assumed that the application of some appropriate stimuli on the dentine surface could extend the changes of fluid movement and activate the pulpal nerves. In 1963, Martin Brännström has published a lot of studies supporting this theory. The studies were performed on both human and animal models. This concept is based on an evidence that the fluid flow in dentinal tubules is stimulus-induced. Thermal, tactile, or chemical stimuli change the movement of fluid and induce deformation of nerve endings in pulp, causing pain [5-7].

There are various methods of $\mathrm{DH}$ treatment. Several of them are based on a decrease of fluid movement due to dentinal tubules sealing or nerve activity blockage. According to publications from recent years, no completely efficient treatment for $\mathrm{DH}$ has been found yet $[3,4,8]$. Propolis, known as the bee-glue, is a natural resinous substance collected from plant buds or exudates by bees. Most often, propolis contains a mixture of resin, essential oils, wax, amino acids, minerals, ethanol, complex of A, E, B vitamins, zinc, pollen, and bioflavonoids also known as the highly active biochemical substance. Biological activity of propolis is mainly associated with well-known plant compounds, such as bioflavonoids. Propolis is widely used not only in medicine, but also in contemporary dentistry because of its anti-inflammatory, anti-oxidant, anti-bacterial, anti-viral, and anti-fungal properties. According to literature review, propolis has been used for DH treatment and showed a significant reduction of pain [9-11].

Many papers have been published on wide usage of $\mathrm{DH}$ laser-based treatment. Variety of types of lasers are recommended for effective DH treatment, of which is a low-level laser therapy (LLLT). In recent studies, the LLLT was suggested as an effective treatment. Low-level laser irradiation increases the metabolic activity of pulp cells, resulting in the production of tertiary dentine [12-14].

In recent years, the most common desensitizer agent was sodium fluoride. A range of desensitizers based on sodium fluoride are recommended for an efficient reduction of pain. The mechanism of action is explained by occluding dentine tubules with calcium fluoride crystals. Moreover, obliteration of dentinal tubules by calcium fluoride crystals reduce the fluid movement. Due to a hydrodynamic theory, this may help to decrease the level of pain $[1,4]$.

\section{OBJECTIVES}

The aim of this study was to compare efficacy of sodium fluoride varnish application, diode laser $(810 \mathrm{~nm})$ irradiation, ethanolic extract of propolis application, and combined diode laser $(810 \mathrm{~nm})$ irradiation with ethanolic extract of propolis application for $\mathrm{DH}$ reduction in periodontal maintenance patients at long-term follow-up.

\section{MATERIAL AND METHODS}

One hundred and twenty patients with periodontal diseases affected by $\mathrm{DH}$ were included into the present study. Patients' recruitment was performed within the Department of Therapeutic Dentistry, Faculty of Dentistry, Kharkiv National Medical University. Inclusion criterion was $\mathrm{DH}$ of teeth in patients with chronic periodontal disease. Exclusion criteria were caries lesions or restorations, any professional DH therapy during the last 6 months, usage of desensitizing toothpaste within the last 3 months, and pregnancy.

Firstly, patients were provided with oral and written information about the intention and design of the study. Secondly, patients were included in our study only after signing an informed consent form. The ethical and bioethical committee of the Kharkiv National Medical University (minutes No. 6 of 4 October 2017) approved the study protocol and related consent forms.

The vitality of all experimental teeth was examined at the beginning and the end of the study by an electric pulp tester (Averon, EOT 1.1, Russia) to exclude pulp pathology. The degree of sensitivity was determined by an examiner using visual analogue scale (VAS). Each tooth response was measured by thermal evaporative stimulus as air blast. A cold air blast was given with an air syringe for 1-2 seconds at approximately $1 \mathrm{~cm}$ at right angle to the buccal site from the surface of tooth. Adjacent teeth were isolated with cotton rolls to prevent false positive results. Air stimulus time was controlled by chronometer, and the distance was measured by a periodontal pocket probe (Surgicon Pvt. Ltd., Pakistan). All patients were requested to record their level of dentine hypersensitivity using VAS scale ranging from 0 to 10 , where 0 was "no pain" and 10 "unbearable pain". A separate sheet of paper with pre-printed VAS scale was provided to patients for their scores. For every assessment of DH level, the patients filled in a new line of VAS to prevent any biased evaluation, which could be influenced by previ- 
ous results. Data from VAS were recorded by measuring the distance between zero point and the patient's record in millimeters on a $10-\mathrm{cm}$ line. One operator provided all stimuli at the same dental chair, with the same equipment, similar air pressure of approximately 55-60 $\Psi$, and temperature of $21-22^{\circ} \mathrm{C}$ at each procedure.

Patients were randomly divided into four groups using Microsoft Office Excel program after the first measurement of DH level, and every group consisted of 30 patients according to $\mathrm{DH}$ treatment. In the first group of patients, diode laser irradiation was used for the treatment of DH. Diode laser (Lika T, Fotonika Plyus, PP, Ukraine) was used with a handpiece with $810 \mathrm{~nm}$ wavelength and $0.1 \mathrm{~W}$ power, $6 \mathrm{~J} / \mathrm{cm}^{2}$ of energy density, in continuous waveform for 180 seconds. The irradiation was performed in a contact mode, perpendicular to the surface, in three points of the cervical area of teeth. At every point, the irradiation lasted for 60 second, with 3 repetition in 48 hours.

In the second group, ethanolic extract of propolis (Ternopharm LLC, Ukraine) was utilized. After removal of debris, sensitive teeth were isolated with cotton rolls and liquid rubber dam (OpalDam, Ultradent, USA) to avoid direct contact with gingiva. After drying tooth surfaces with a cotton pellet, extract of propolis was applied using a rubber cup in slow speed handpiece (FPB-EC Push Button Contra Angle Handpiece, NSK, Japan) for 30 seconds and let it to dry for 60 seconds, paying particular attention that desensitizing agent did not touch any area of oral mucosa. The procedure was performed 3 times for 3 days.

In the third group of patients, a combination of the two above-mentioned techniques was used. At first, application of ethanolic extract of propolis was done, followed by laser irradiation. Application of desensitizing agent and laser irradiation were the same as in groups 1 and 2 . The procedure was repeated three times in every 48 hours.

In the fourth group, sodium fluoride varnish was applied. Application of ftorplen (Latus, Ukraine) was completed as per manufacturer's recommendations. After debris removal, the buccal surface of sensitive teeth was dried and isolated with cotton rolls. Sodium fluoride was applied using an applicator. The tooth surface was dried with an air syringe for 30 seconds. The procedure was repeated three days in a row.

The treatment was performed for all $\mathrm{DH}$ teeth, with one procedure per patient in all groups at the beginning of our study. For the evaluation of results, only one tooth with $\mathrm{DH}$ was randomly chosen in every quadrant (four teeth for one patient). Values were collected before the treatment (baseline values), and the included teeth were evaluated after the procedure (for immediate assessment of results), at one week, one month, three months, and six months post-operatively. The patients were provided with instructions, such as not to rinse, eat, or drink for 30 minutes after the treat- ment, and to avoid using any other desensitizing agent during the study.

All data were collected using Microsoft Excel sheet. Statistical analysis was performed by IBM SPSS Statistics for Windows, version 25.0 (IBM Corp., Armonk, NY, USA). Firstly, mean and standard deviation were calculated for each group. Since the data were not distributed normally, non-parametric tests were selected. Wilcoxon's rank sum test was used to evaluate the differences within groups at each time point. Intragroup time-dependent data were analyzed by Friedman's test. Values of $p<0.05$ were considered as statistically significant.

\section{RESULTS}

Of 120 patients included in our study, 104 completed the 6-month study period. There were 34 males and 70 females, with a mean age of $40.91 \pm 9.03$ years. Mean age and standard deviation in all groups are presented in Table 1. Due to reduced number of patients who completed the full study, the number of patients in each group became 26 . The total number of teeth included in our study was 416 . Table 2 presents teeth distribution.

Complications such as adverse pulp effects or allergic reactions were not observed throughout the study. Responses of the patients to thermal evaporative stimulus by visual analogue scale (VAS) throughout the study and the effects of treatments in the four groups at different time points are shown in Figure 1.

TABLE 1. Mean and standard deviation of patients' age

\begin{tabular}{|l|c|}
\hline Group & Age \\
\hline 1 & $40.38 \pm 9.37$ \\
\hline 2 & $40.81 \pm 9.24$ \\
\hline 3 & $41.04 \pm 9.04$ \\
\hline 4 & $41.42 \pm 8.98$ \\
\hline
\end{tabular}

TABLE 2. Distribution of teeth included in study

\begin{tabular}{|l|c|}
\hline Teeth & Number of teeth \\
\hline Maxillary central incisors & 25 \\
\hline Maxillary lateral incisors & 24 \\
\hline Maxillary canine & 51 \\
\hline Maxillary premolars & 81 \\
\hline Maxillary molars & 27 \\
\hline Mandibular central incisors & 26 \\
\hline Mandibular lateral incisors & 26 \\
\hline Mandibular canine & 55 \\
\hline Mandibular premolars & 76 \\
\hline Mandibular molars & 25 \\
\hline
\end{tabular}


TABLE 3. Mean and standard deviation of thermal evaporative stimulus scores by VAS

\begin{tabular}{|l|c|c|c|c|c|c|}
\hline Group & Baseline & Immediate & Week 1 & Month 1 & Month 3 & Month 6 \\
\hline 1 & $6.97 \pm 0.76$ & $1.70 \pm 0.46^{*}$ & $1.93 \pm 0.25^{*}$ & $2.19 \pm 0.39^{*}$ & $2.36 \pm 0.48^{*}$ & $2.49 \pm 0.50^{*}$ \\
\hline 2 & $6.89 \pm 0.81$ & $1.91 \pm 0.29^{*}$ & $2.18 \pm 0.38^{*}$ & $2.88 \pm 0.69^{*}$ & $3.44 \pm 0.51^{*}$ & $3.82 \pm 0.59^{*}$ \\
\hline 3 & $6.92 \pm 0.78$ & $1.51 \pm 0.50^{*}$ & $1.68 \pm 0.46^{*}$ & $1.93 \pm 0.25^{*}$ & $1.71 \pm 0.45^{*}$ & $1.43 \pm 0.49^{*}$ \\
\hline 4 & $6.79 \pm 0.73$ & $1.61 \pm 0.49^{*}$ & $1.48 \pm 0.50^{*}$ & $2.08 \pm 0.27^{*}$ & $2.85 \pm 0.35^{*}$ & $3.58 \pm 0.58^{*}$ \\
\hline
\end{tabular}

Mean VAS scores and standard deviations at different times of the study in the four groups are demonstrated in Table 3. Based on intragroup comparisons, the treatment of DH was significantly effective in all four groups (Table 3). Intragroup comparisons showed that the differences between baseline scores and immediate, 1 week, 1,3 , and 6 months after the treatment were statistically significant $(p<0.05)$. In all four groups, immediate responses for thermal evaporative stimulus showed a significant reduction of level of pain. Responses in group 3 revealed significant differences among the scores of baselines, immediately after the treatment, and at 1 week, 1,3 , and 6 months post-operatively (Table 3).

Paired comparisons showed significant differences between the treatment groups, with $p<0.05$. No significant differences between the treatment groups were found at baseline $(p>0.05)$. Additionally, no significant differences between group 1 and 4 , and group 3 and 4 for thermal evaporative stimulus immediately after the treatment were observed.

Comparison between groups showed that the lowest VAS scores for thermal evaporative stimulus were obtained in group 3 during the study (Figure 1).

\section{DISCUSSION}

$\mathrm{DH}$ is a common condition for patients with periodontal disease, because of exposed root surfaces and gingival recession. However, with multiple variants of treatments, there is still no gold standard for reducing $\mathrm{DH}[4,8]$. The present clinical study compares the efficacy of sodium fluoride varnish, ethanolic extract of propolis application, diode laser $(810 \mathrm{~nm})$ irradiation, and the combination of two methods, such as application of ethanolic extract of propolis and diode laser $(810 \mathrm{~nm})$ irradiation in decreasing the symptoms of $\mathrm{DH}$.

Patient's pain and discomfort caused by dentine hypersensitivity is individual and highly subjective. In most studies, scales are used for assessment of $\mathrm{DH}$. The most frequent are visual analogue scale (VAS) and Schiff sensitivity scale [4]. According to literature review, scientists evaluated $\mathrm{DH}$ with the use of thermal evaporative stimuli, such as cold air blast provided with an air syringe. Findings of these studies demonstrated that evaporative stimuli dehydrated the dentine surface,

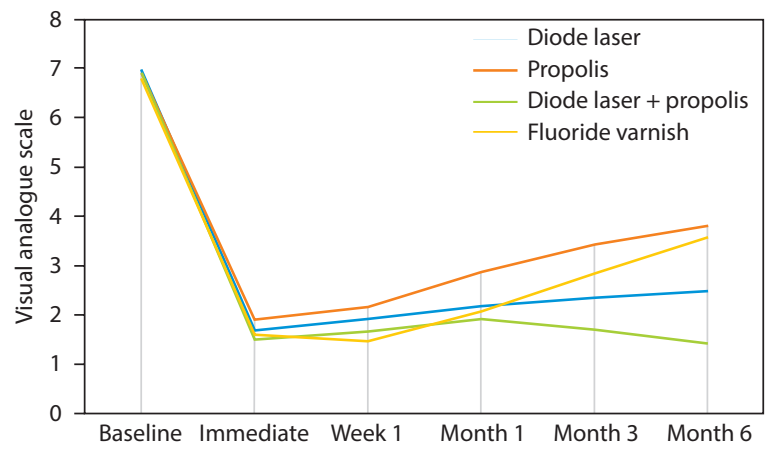

FIGURE 1. Reduction of dentine hypersensitivity in all studied groups (thermal evaporative stimulus)

which increased the movement of fluid in dentine tubules [6].

In our study, sodium fluoride varnish was used, because sodium fluoride is the most frequent agent of desensitizers and we sought to compare its efficiency with usefulness of our design of $\mathrm{DH}$ treatment. The results of our study are in accordance with other researches and confirm that application of sodium fluoride desensitizer shows immediate result in the reduction of $\mathrm{DH}-$ related pain, however, with a short-term effect $[4,12]$. The 6 months VAS scores did not return to the baseline values but were significantly higher when compared to a combined treatment.

Here, ethanolic extract of propolis was chosen as a natural desensitizing agent and because of the occurrence in many studies, in which showed a high efficacy in DH treatment [15-19]. In our study, the immediate VAS scores were significantly lower than the baseline values. However, during the period of study, the meaning of VAS scores increased. Studies on propolis comparing other desensitizers provided significant results in reducing $\mathrm{DH}[2,20]$. In the present study, a significant difference between the baseline and 6-month VAS scores was also obtained.

In recent times, laser irradiation was proven efficient for DH treatment [21-24]. In our study, diode laser was chosen as an LLLT appliance, and it was successfully implemented. Many studies reported the same results. However, most of them described a higher decrease in the 6-month VAS scores, which was not obtained 
in our study. It could be explained that all these studies were performed using different diode lasers, with a wide range of wavelengths of 635-830 $\mathrm{nm}$ and dosages of $2-10 \mathrm{~J} / \mathrm{cm}^{2}$. The usage of all these parameters is acceptable and does not affect the morphology of enamel or dentine surface [12-14, 21-24].

According to literature review, in this study, we wanted to combine all the impacts of application of propolis ethanolic extract with diode $(810 \mathrm{~nm})$ laser irradiation for $\mathrm{DH}$ treatment. We considered that this design of $\mathrm{DH}$ treatment can improve and prolong the outcomes in decreasing the level of pain.

The mechanism of action of both methods are not completely clear. For example, scientists observed that the dentine binds to propolis flavonoids and forms crystals with an ability to adhere to the dentine surface. As a result, the dentine tubules are occluded. On the other hand, propolis consists of various natural resinous substances. It is confirmed that propolis could be resemble dental adhesive substances as varnishes or composite resins. The dentine tubules are occluded because mechanical interlocking could prevent fluid flow. Moreover, propolis may deeply diffuse inside dentinal tubules and be difficult to remove, which could sufficiently prolong the result of treatment [15-17]. However, the mechanism of action of propolis requires more studies for clarity. Other studies showed that the production of transforming growth factor (TGF)- $\beta 1$ is stimulated by propolis. Moreover, TGF- $\beta 1$ is important for odontoblast-like cell differentiation. According to studies, in a case of direct pulp-capping, propolis paste partial dentine bridge formation was observed after four weeks. This is explained by reparative dentinogenesis stimulated by propolis flavonoids, which can product tertiary dentine and affect dentinal fluid movement $[18,19]$.

LLLT or photobiomodulation (PBM) is known for using light energy to elicit biological responses from the cell and to normalize its function $[25,26]$. A small fraction of laser energy at $810 \mathrm{~nm}$ wavelength reaches the pulp and transmits through the dental hard tissues. Therefore, the physiological cellular functions may normally be stimulated by LLLT. In addition, laser energy may promote the production of sclerotic dentine and the internal occlusion of dentine tubules. However, the results of in-vitro studies revealed that even such a small dosage could melt the dentine, producing a double-layer structures of tubules. Therefore, narrowing of the dentine tubules decreases the internal fluid movement, which could explain immediate analgesic effect caused by depressing nerve transmission that stops the C-fiber afferents depolarization $[13,14]$.

The present study was designed based on the abovementioned facts. Even though our research showed good results in reducing $\mathrm{DH}$, experimental studies are still necessary for understanding the exact mechanism of suggested type of DH treatment.

\section{CONCLUSIONS}

In our study, the efficacy of four types of DH treatment was assessed. All groups of patients showed statistically significant results. The highest results were obtained after a combined DH treatment. Therefore, combined application of ethanolic extract of propolis with diode laser $(810 \mathrm{~nm})$ irradiation can be recommended for $\mathrm{DH}$ treatment because of immediate and prolong reduction of the level of pain without any adverse reactions.

\section{CONFLICT OF INTEREST}

The authors declare no potential conflicts of interest with respect to the research, authorship, and/or publication of this article.

\section{References}

1. Canadian Advisory Board on Dentin Hypersensitivity. Consensus-based recommendations for the diagnosis and management of dentin hypersensitivity. J Can Dent Assoc 2003; 69: 221-226.

2. Arjun Torwane N, Hongal S, Goel P, et al. Effect of two desensitizing agents in reducing dentin hypersensitivity: an in-vivo comparative clinical trial. J Clin Diagnostic Res 2013; 7: 2042-2046.

3. Splieth $\mathrm{CH}$, Tachou A. Epidemiology of dentin hypersensitivity. Clin Oral Investig 2013; 17: 3-8.

4. Benoist L, Niang F, Faye SO, Sarr B, Seck M. Treatment of dentin hypersensitivity: a systematic review of randomized clinical trials. J Dent Oral Care Med 2016; 2: 204.

5. Ali S, Farooq I. Dentin hypersensitivity: a review of its etiology, mechanism, prevention strategies and recent advancements in its management. World J Dent 2013; 4: 188-192.

6. West NX, Lussi A, Seong J, Hellwig E. Dentin hypersensitivity: pain mechanisms and aetiology of exposed cervical dentin. Clin Oral Investig 2013; 17: 9-19.

7. Brännström M, Äström A. The hydrodynamics of the dentine; its possible relationship to dentinal pain. Int Dent J 1972; 22: 219 227.

8. Porto IC, Andrade AK, Montes MA. Diagnosis and treatment of dentinal hypersensitivity. J Oral Sci 2009; 51: 323-332.

9. Khurshid Z, Naseem M, Zafar MS, Najeeb S, Zohaib S. Propolis: a natural biomaterial for dental and oral healthcare. J Dent Res Dent Clin Dent Prospects 2017; 11: 265-274.

10. Abbasi AJ, Mohammadi F, Bayat M, Gema SM, Ghadirian H, Seifi H, Bahrami N. Applications of propolis in dentistry: a review. Ethiop J Health Sci 2018; 28: 505-512.

11. Więckiewicz W, Miernik M, Więckiewicz M, Morawiec T. Does propolis help to maintain oral health? Evid Based Complement Alternat Med 2013; 2013: 351062.

12. Yilmaz HG, Kurtulmus-Yilmaz S, Cengiz E. Long-term effect of diode laser irradiation compared to sodium fluoride varnish in the treatment of dentine hypersensitivity in periodontal maintenance patients: a randomized controlled clinical study. Photomed Laser Surg 2011; 29: 721-725.

13. Kimura Y, Wilder-Smith P, Yonaga K, Matsumoto K. Treatment of dentine hyper-sensitivity by lasers: a review. J Clin Periodontol 2000; 27: 715-721.

14. Biagi R, Cossellu G, Sarcina M, Pizzamiglio IT, Farronato G, Cà Granda F. Laser-assisted treatment of dentinal hypersensitivity: a literature review. Ann Stomatol (Roma) 2016; 6: 75-80.

15. Chen CL, Parolia A, Pau A, Celerino De Moraes Porto IC. Comparative evaluation of the effectiveness of desensitizing agents in 
dentine tubule occlusion using scanning electron microscopy. Aust Dent J 2015; 60: 65-72.

16. Almas K, Mahmoud A, Dahlan A. A comparative study of propolis and saline application on human dentin. A SEM study. Indian J Dent Res 2001; 12: 21-27.

17. Sales-Peres SH, Carvalho FN, Marsicano JA, et al. Effect of propolis gel on the in vitro reduction of dentin permeability. J Appl Oral Sci 2011; 19: 318-323.

18. Sabir A, Tabbu CR, Agustiono P, Sosroseno W. Histological analysis of rat dental pulp tissue capped with propolis. J Oral Sci 2005; 47: 135-138

19. Parolia A, Kundabala M, Rao NN, et al. A comparative histological analysis of human pulp following direct pulp capping with propolis, mineral trioxide aggregate and Dycal. Aust Dent J 2010; 55: 59-64.

20. Hongal S, Torwane NA, Goel P, Chandrashekar B. The effect of $30 \%$ ethanolic extract of indian propolis on replica of human dentin compared against commercially available desensitizing agent: a methodological SEM study in vitro. Pharmacognosy Res 2014; 6: 113-119.

21. Hashim NT, Gasmalla BG, Sabahelkheir AH, Awooda AM. Effect of the clinical application of the diode laser $(810 \mathrm{~nm})$ in the treatment of dentine hypersensitivity. BMC Res Notes 2014; 7: 31.

22. Pandey R, Koppolu P, Kalakonda B, et al. Treatment of dentinal hypersensitivity using low-level laser therapy and 5\% potassium nitrate: a randomized, controlled, three arm parallel clinical study. Int J Appl Basic Med Res 2017; 7: 63-66.

23. Lopes AO, Aranha ACC. Comparative evaluation of the effects of Nd:YAG laser and a desensitizer agent on the treatment of dentin hypersensitivity: a clinical study. Photomed Laser Surg 2013; 31: 132-138.

24. Yilmaz HG, Kurtulmus-Yilmaz S, Cengiz E, Bayindir H, Aykac Y. Clinical evaluation of Er,Cr:YSGG and GaAlAs laser therapy for treating dentine hypersensitivity: a randomized controlled clinical trial. J Dent 2011; 39: 249-254

25. Karu TI. Multiple roles of cytochrome c oxidase in mammalian cells under action of red and IR-A radiation. IUBMB Life 2010 62: 607-610.

26. Ross G. Photobiomodulation in dentistry. Photomed Laser Surg 2012; 30: 565-567. 